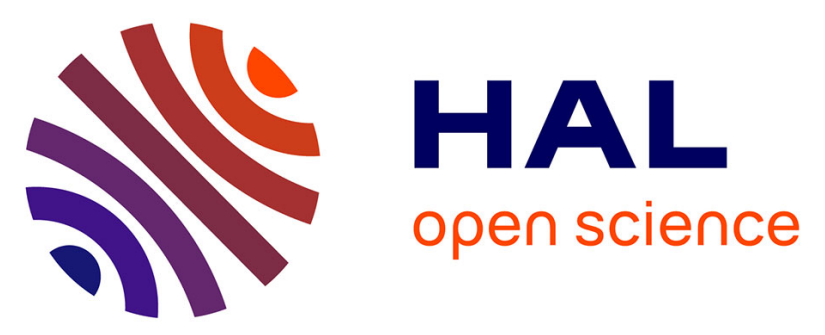

\title{
A novel mutation in the dynamin 2 gene in a Charcot-Marie-Tooth type 2 patient: Clinical and pathological findings
}

Marc Bitoun, Tanya Stojkovic, Bernard Prudhon, Claude-Alain Maurage, Philippe Latour, Patrick Vermersch, Pascale Guicheney

\section{To cite this version:}

Marc Bitoun, Tanya Stojkovic, Bernard Prudhon, Claude-Alain Maurage, Philippe Latour, et al.. A novel mutation in the dynamin 2 gene in a Charcot-Marie-Tooth type 2 patient: Clinical and pathological findings. Neuromuscular Disorders, 2008, 18 (4), pp.334-338. 10.1016/j.nmd.2008.01.005 . hal-02448631

\section{HAL Id: hal-02448631 \\ https://hal.science/hal-02448631}

Submitted on 22 Jan 2020

HAL is a multi-disciplinary open access archive for the deposit and dissemination of scientific research documents, whether they are published or not. The documents may come from teaching and research institutions in France or abroad, or from public or private research centers.
L'archive ouverte pluridisciplinaire HAL, est destinée au dépôt et à la diffusion de documents scientifiques de niveau recherche, publiés ou non, émanant des établissements d'enseignement et de recherche français ou étrangers, des laboratoires publics ou privés. 


\section{A novel mutation in the dynamin 2 gene in a Charcot-Marie-Tooth type 2 patient: clinical and pathological findings}

Marc Bitoun $^{1,2^{*}}$, Tanya Stojkovic ${ }^{3 *}$, Bernard Prudhon ${ }^{1,2}$, Claude-Alain Maurage ${ }^{4}$, Philippe Latour $^{5}$, Patrick Vermersch ${ }^{6}$, Pascale Guicheney ${ }^{1,2,7}$

1. INSERM, U582, Institut de Myologie, Paris, France.

2. Université Pierre et Marie Curie-Paris6, UMR S582, IFR14, Paris, France.

3. Centre de Référence de Pathologie Neuromusculaire Paris-Est, Groupe Hospitalier PitiéSalpêtrière, Paris, France.

4. Service de Neuropathologie, CHU de Lille, Lille, France.

5. Service de Neurogénétique Moléculaire, Centre Hospitalier Est, Bron, France

6. Pôle de Neurologie, Centre de référence des maladies Neuromusculaires Nord, CHU de Lille, Lille, France.

7. AP-HP, Groupe Hospitalier Pitié-Salpêtrière, Paris, France.

* These authors are considered as equal first co-authors.

Running title: Clinical and pathological findings in DNM2-CMT.

\section{Corresponding Author:}

Dr. Marc Bitoun, Inserm U582, Institut de Myologie, Groupe Hospitalier Pitié-Salpêtrière, 75013, Paris, France.

E-mail: m.bitoun@myologie.chups.jussieu.fr

Tel : $33(0)$ 1.42.16.57.35

Fax: $33(0)$ 1.42.16.57.00 
Title: A novel mutation in the Dynamin 2 gene in a Charcot-Marie-Tooth type 2 patient: clinical and pathological findings

\begin{abstract}
:
Mutations in Dynamin 2 (DNM2) have been associated with autosomal dominant centronuclear myopathy, dominant intermediate Charcot-Marie-Tooth (CMT) type B and CMT2. Here, we report a novel DNM2 mutation in the Pleckstrin homology domain of DNM2 (p.K559del) in a patient with an axonal length-dependent sensorimotor polyneuropathy predominantly affecting the lower limbs. Neuropathy is associated with congenital cataracts, ophthalmoparesis, ptosis and neutropenia. There was no evidence of a skeletal myopathy on EMG or muscle biopsy. We suggest that this constellation of clinical features can help the diagnosis and selection of patients for direct DNM2 genetic analysis.
\end{abstract}

Keywords: Charcot-Marie-Tooth neuropathy, Dynamin 2 mutation, clinical findings, muscle biopsy. 


\section{Introduction.}

Dynamin 2 (DNM2) mutations have been associated with two distinct clinical presentations: autosomal dominant centronuclear myopathy (CNM) [1,2] and dominant intermediate and axonal Charcot-Marie-Tooth disease (CMT) [3,4]. DNM2-CNM is a slowly progressive congenital myopathy characterized by frequent centrally located nuclei in muscle fibres. Muscle weakness is often associated with bilateral ptosis and ophthalmoplegia [5]. The motor nerve conduction velocities (MNCV) in DNM2-CMT are variable, so that patients may have features of the intermediate form of CMT (MNCV between $25 \mathrm{~m} / \mathrm{s}$ and $38 \mathrm{~m} / \mathrm{s}$ ) or the axonal form with MNCV above $38 \mathrm{~m} / \mathrm{s}$. In addition to distal muscle weakness and sensory impairment that is common to all forms of CMT, neutropenia has also been identified in some DNM2-CMT patients [3].

Mild axonal peripheral nerve involvement in addition to the predominant myopathic changes was reported in some DNM2-CNM patients [5]. In contrast, the presence of myopathic features in DNM2-CMT patients has not been reported. In a recent study of three DNM2-CMT patients, no myopathic changes were observed on electromyography (EMG) or magnetic resonance imaging (MRI) [4] but, these patients did not undergo muscle biopsy. Here, we reported the clinical and muscle biopsy findings in a CMT-patient harbouring a novel DNM2 mutation in the Pleckstrin homology $(\mathrm{PH})$ domain. 


\section{Case report.}

\subsection{Clinical history}

The patient is the second child of a family without history of neuromuscular disorders. After a normal prenatal period, she presented at birth with a congenital convergent strabismus and asymmetric ptosis. Bilateral nuclear cataracts were detected at 1 year. She had normal motor development and walked independently from age 16 months although she had difficulties in jumping, running and had recurrent ankle sprains.

She underwent corrective surgery for ptosis and strabismus due to abducens muscle palsy at the ages of 4 and 6 years. At that time, her visual acuity was $1 / 10$ and 4/10 for the left and right eyes, respectively. She had progressive weakness of foot extensors that lead to walking difficulties but she was still ambulant at age 14 years. Neurological examination revealed wasting of intrinsinc foot muscles and milder atrophy of interosseous and extensor muscles of the hand. There was weakness of toes and foot dorsiflexors, graded respectively as 2 and 3 on the Medical Research Coucil (MRC) rating scale for muscle strength. The peroneus muscles and the muscles of plantar flexion were graded as 3 bilaterally. In the upper limbs, the interosseous and extensor muscles of the hand were graded 3 on MRC score, whereas the thenar and hypothenar muscles were preserved. There was no proximal muscle weakness and no scapular winging. She had also bilateral pes cavus and claw toes. Pain, light touch, vibration and joint position perception were reduced below the knee. Deep tendon reflexes were abolished in the lower limbs and present in the upper limbs. In addition, she had mild bilateral ptosis and limited abduction of both eyes.

Nerve conduction studies performed at age 15 years showed mildly reduced median and ulnar nerve motor conduction velocities $(45 \mathrm{~m} / \mathrm{s}$ and $44 \mathrm{~m} / \mathrm{s}$ respectively; normal >48 
$\mathrm{m} / \mathrm{s}$ ), and normal distal latencies of $3.5 \mathrm{~ms}$ (median nerve) and $2.5 \mathrm{~ms}$ (ulnar; normal <3.5). There were normal median $(9 \mathrm{mV})$ and ulnar $(7 \mathrm{mV})$ compound muscle action potential (CMAP) amplitudes (normal $>5 \mathrm{mV}$ ). In the lower limbs, stimulation of the peroneal and posterior tibial nerves evoked no motor responses when recorded from the extensor digitorum brevis and abductor hallucis muscles. The motor nerve conduction of the peroneal nerve was normal, when recorded from anterior tibialis (CMAP: $3.5 \mathrm{mV}$, normal $>2 \mathrm{mV}$, MNCV: 44 $\mathrm{m} / \mathrm{s}$, normal $>42 \mathrm{~m} / \mathrm{s}$ ). Sensory nerve amplitudes (SNAP) were abolished in the lower limbs and diminished in the upper limbs. Median and ulnar SNAP respectively were $12 \mu \mathrm{V}$ (normal $>15 \mu \mathrm{V}$ ) and $7 \mu \mathrm{V}$ (normal $>8 \mu \mathrm{V}$ ). EMG showed a neurogenic pattern in the distal muscles of the four limbs. These results were in favour of an axonal length-dependent sensorimotor polyneuropathy predominantly affecting the lower limbs.

Electrocardiogram, echocardiogram and brain MRI were normal. In addition to the nuclear cataracts detected at age 1 year, ophthalmologic examination at age 2 years showed the presence of a lateral gaze palsy and mild ptosis. The patient's electroretinogram was normal and visual acuity was stable at the age of 15 years: $1 / 10$ and $5 / 10$ for the left and right eye, respectively. The reduced visual acuity in the left eye was assumed to be due to amblyopia. A low neutrophil count of $750 / \mathrm{mm}^{3}$ (normal $>1500 / \mathrm{mm}^{3}$ ) had been documented since the first year of age. Platelet and erythrocytes were in the normal range.

\subsection{Muscle biopsy findings.}

A muscle biopsy was taken from the deltoid muscle at age 15 years and analysed using standard histochemical stains (Figure 1). There was no nuclear internalization on HemateinEosin staining. G3PDH staining showed a normal ratio of type 1 and 2 fibres and some angulated fibers of both types. The intermyofibrillary network appeared almost normal on 
NADH-TR staining with a slight subsarcolemmal accumulation of oxidative activity. Respiratory chain enzyme activity was in the normal range for age in the muscle biopsy. No mitochondrial DNA mutations or deletions were detected. No abnormalities were identified on other routine histochemical stains (Gomori trichrome, Periodic acid-Schiff, Oil red O, acid and alkaline phosphatases, succinic dehydrogenase and cytochrome oxidase). Electron microscopy showed absence of morphological abnormalities (Figure 1D).

\subsection{Mutation analysis.}

DNA from the patient and her parents was extracted from blood samples and all the exons of DNM2 gene were sequenced using methods previously reported [1]. We identified a heterozygous three base-pair deletion located in exon 15 of DNM2 in the patient (Figure 2). The deletion (c.1675_1677delAAA) results in the loss of the highly conserved lysine 559 (p.K559del) located in the PH domain. The mutation was not found in either parent, consistent with a de novo occurrence in the patient and was not found in 100 unrelated healthy subjects. No mutation was found in the genes encoding Myelin Protein Zero, Peripheral Myelin Protein 22 or Connexin 32. 


\section{Discussion.}

We report a novel DNM2 mutation in a patient with a sensorimotor peripheral neuropathy associated with congenital cataracts, ophthalmoparesis, ptosis and neutropenia. Electrophysiological data was typical of axonal CMT (CMT2) in agreement with the recent report from Fabrizi et al. [4] but contrasting with the initial report indicating intermediate value of MNCV in patients with DNM2 mutations [3]. Taken together, these results suggest that MNCV may vary widely in the DNM2-CMT, even in the same family, probably depending of the stage and severity of the disease.

With the present report included, DNM2 mutations have been found in patients from six CMT families $[3,4]$. The clinical spectrum in these families is wide, from mild and slowly progressive CMT to a more severe form with proximal and distal weakness and more rapid disease progression. Neutropenia was reported in 3 out of 6 families, indicating that this is a variable feature in DNM2-CMT. Our patient had ptosis, ophthalmoparesis and cataract. Ptosis and ophthalmoparesis have been frequently associated with DNM2 mutations in CNM. We show that these abnormalities can also be associated with DNM2-CMT possibly resulting from muscle involvement or cranial nerve abnormalities as already described in rare CMT patients [6]. Cataract has previously been reported in CNM [7] but, to date, there is no report illustrating the presence of cataract in either DNM2-CNM or DNM2-CMT. However, some DNM2-CMT patients have cataract in the previously reported Belgian family [3, Kristl Claeys, personal communication). In the patient we report, a coincidental association between the DNM2 mutation and cataract cannot be excluded in spite of absence of other known causes of cataract (no metabolic disorders, infectious diseases or intake of teratogen during pregnancy). Identification and clinical description of additional DNM2-CMT patients will be necessary to confirm a genuine association between the ptosis, ophthalmoparesis and cataracts 
and CMT-DNM2 mutations that we report. In light of the genetic and clinical heterogeneity in CMT, recognition of such an association is likely to aide the diagnosis of DNM2-related CMT.

DNM2 is a ubiquitous large GTPase that has been implicated in endocytosis and more recently in centrosome organization [8]. DNM2 is composed of several domains, including a Pleckstrin Homology $(\mathrm{PH})$ domain which is classically involved in targeting proteins to the plasma membrane, through phosphoinositides binding. All the CMT mutations reported to date affect residues in this domain $[3,4]$. The deletion reported here leads to a deletion of a highly conserved lysine 559, also located in the PH domain. One can hypothesise that these CMT mutations modify the three-dimensional structure of the $\mathrm{PH}$ domain resulting in a decrease in membrane trafficking. In addition, since DNM2 is a component of the centrosome [8], the main microtubule organizing center, the CMT mutations could induce a destabilization of the microtubule network leading to abnormal axonal transport and protein trafficking, a pathophysiological mechanism described in various forms of CMT [9].

One important unresolved issue has been to determine whether a myopathy co-exists with the neuropathy in DNM2-CMT patients. A recent study found no myopathic changes in three DNM2-CMT patients [4] but patients did not undergo muscle biopsy. We found no myopathic changes on EMG or muscle biopsy in our patient, in addition to the typical electrophysiological findings of CMT. In particular, the morphological features of DNM2CNM were absent i.e. centrally located nuclei, radial oriented sarcoplasmic strands and predominance and hypotrophy of type 1 fibres. The age at biopsy cannot explain the absence of muscle abnormalities, since in CNM patients the morphological changes are typically present in the deltoid muscle at age 15 years [10]. These results show that some DNM2 mutations are associated with nearly normal muscle histology and further studies will be 
necessary to explain the differential effects of DNM2 mutations in CNM and CMT on muscle histology.

In summary, we report a novel DNM2 mutation leading to CMT disease associated with congenital cataract, ptosis, ophthalmoparesis and neutropenia, features that are not classically observed in CMT disease. The identification of this particular clinical phenotype may be helpful for diagnosis and to orient genetic analysis toward DNM2. 


\section{References}

[1] Bitoun M, Maugenre S, Jeannet $\mathrm{P}$, et al. Mutations in dynamin 2 cause dominant Centronuclear Myopathy. Nat Genet 2005;37:1207-1209.

[2] Bitoun M, Bevilacqua JA, Prudhon B, et al. Dynamin 2 mutations cause sporadic centronuclear myopathy with neonatal onset. Ann Neurol. 2007;In press.

[3] Züchner S, Noureddine M, Kennerson M, et al. Mutations in the pleckstrin homology domain of dynamin 2 cause dominant intermediate Charcot-Marie-Tooth disease. Nat Genet 2005;37:289-294.

[4] Fabrizi GM, Ferrarini M, Cavallaro T, et al. Two novel mutations in dynamin-2 cause axonal Charcot-Marie-Tooth disease. Neurology 2007;69:291-295.

[5] Fischer D, Herasse M, Bitoun M, et al. Characterization of the muscle involvement in dynamin 2 related centronuclear myopathy. Brain 2006;129:1463-1469.

[6] Parman, Y. Hereditary neuropathies Curr Opin Neurol 2007;20:542-547.

[7] Vallat JM, Hugon J, Fressinaud C, et al. Centronuclear myopathy, cataract, and electrical myotonia: a new case. Muscle Nerve 1985;8:807-809.

[8] Thompson HM, Cao H, Chen J, et al. Dynamin 2 binds gamma-tubulin and participates in centrosome cohesion. Nat Cell Biol 2004;6:335-342. 
[9] Shy ME. Charcot-Marie-Tooth disease: an update. Curr Opin Neurol 2004;17:579-585.

[10] Fischer D, Herasse M, Bitoun M, et al. Characterization of the muscle involvement in dynamin 2 related centronuclear myopathy. Brain 2006;129:1463-1469. 


\section{Acknowledgements}

We thank the patient and her family for their participation in this study, Dr N Clarke for helpful advices and the Institut National de la Santé et de la Recherche Médicale (INSERM) and the Association Française contre les Myopathies (AFM) for financial support. Marc Bitoun was a recipient of an INSERM fellowship. 


\section{Legends}

\section{Figure 1: Muscle biopsy findings}

Representative fields of Hematein-Eosin (A), Nicotinamide Adenine DinucleotideTetrazolium Reductase (B) and Glyceraldehyde-3-phosphate-dehydrogenase (C) staining and electron microscopy (D) on the muscle biopsy from the patient (deltoid biopsy at the age of 15 years). Structure and organization of muscle fibers were mostly conserved. The only abnormalities were slight sub-sarcolemmal accumulation of oxidative activity and the presence of some angulated fibers.

\section{Figure 2: DNM2 mutation and multiple protein alignment}

A. Electrophoregrams of $D N M 2$ exon 15 showing wild type sequence in an unaffected subject and the heterozygous mutation identified in the patient. The mutation results in the deletion of a lysine residue at position 559. Nucleotides were numbered according to isoform $1 \mathrm{cDNA}$ (GenBank accession number NM 001005360). B. Position of the DNM2-CMT mutations on multiple protein alignment. Amino-acids were numbered according to the isoform 1 (GenBank accession number: NP_001005360) which includes the four amino-acids GEIL at positions $516-519$ encoded by exon 13 bis. Previously reported mutations $[3,4]$ are indicated in green and the novel mutation is indicated in red. All mutations affect residues conserved in the dynamin family of genes in humans and in orthologues from several species. 
14- Clinical and pathological findings in DNM2-CMT
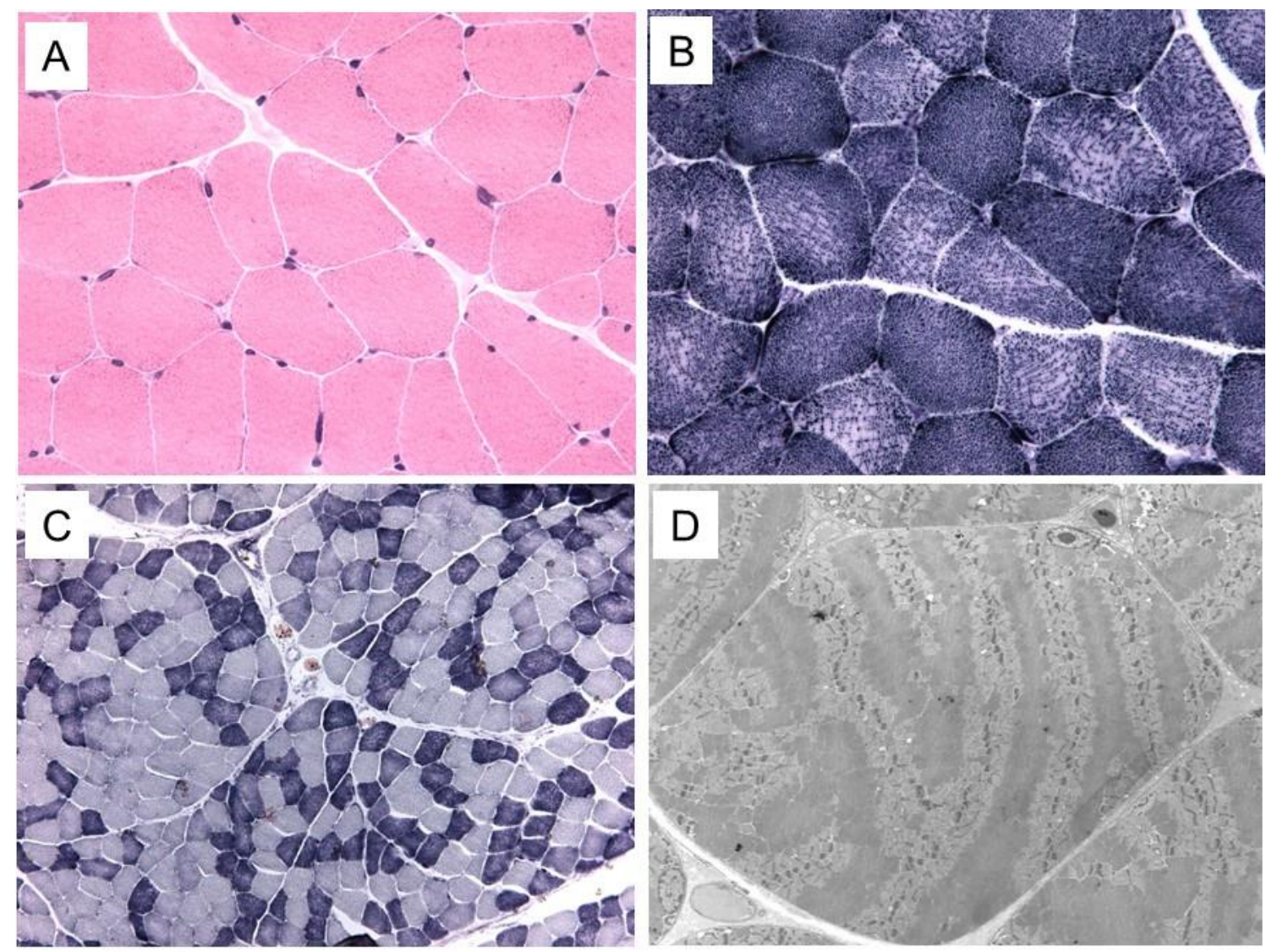

Clinical and pathological findings in DNM2-CMT - Figure 1 
A $\quad$ c.1675-1677del

p. $K 559 \mathrm{del}$

GTACTTCTTTTTTTTCTGAGGGGGGAGGA

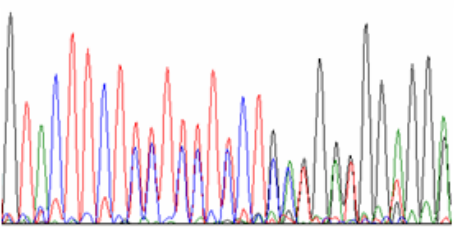

B amino-acid
Healthy Control

GTACTTCTTCTCTTTCTCCTGAGGGTGGAGG

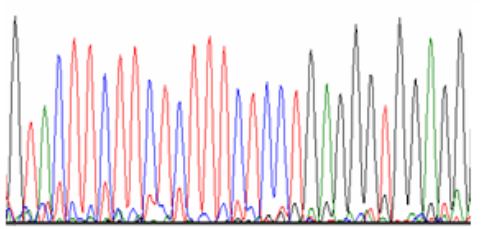

DNM2 Human

DNM2 Mouse

DNM1 Human

DNM3 Human

DNM E $\overline{\text { E ugu }}$

LMKGGSKEYTWEVLT AE S LSWYKDEE E KE KKYMLPLDN LKIRD LMKGGSKEYWFVLT AE S LSWYKDEEE KE KKYMLPLDNLKIRD IMKGGSKEYTWVLT AENLSWYKDDEE KE KKYMLSVDNLKLRD IMKGGSKGYTWVLT AES LSWYKDDEE KE KKYMLPLDNLKVRD IMKGGSKEYTWFVLT AE S LSWYKDEEE KE KKYMLPLDNLKIRD FVRG-SKDNWFVLM SDS LSWYKDDE E KE KKYMLPLDGVKLKD

Figure 2 